\title{
Multi-Gluon Production at High Energies
}

\author{
Michael Lublinsky \\ State University of New York - Department of Physics and Astronomy \\ Stony Brook NY 11794-3800, USA
}

\begin{abstract}
A systematic approach towards description of semi-inclusive processes at low $x$ and with multiple rescatterings taken into account is highlighted. We solve the problem of inclusive multi-gluon production for arbitrary number of gluons, thus extending previously known results for one and two gluons produced.
\end{abstract}

This talk is based on Refs. [1].

Within the BFKL approximation the problem of multi-gluon production is solved (Fig. 1) in terms of the BFKL Green function $G^{B F K L}$ and the effective vertex for gluon emission $L(k)$ with $k$ being a momentum of emitted gluon. A schematic expression for the cross section of $n$-gluon production emitted at different rapidities $Y_{1} \ldots Y_{n}$ and having momenta $k_{1} \ldots k_{n}$ reads (with $\Phi^{P, T}$ standing for projectile/target impact factor)
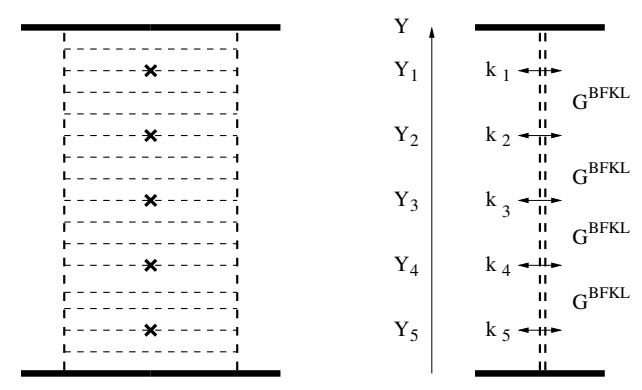

Figure 1: Multi-gluon production within BFKL

$$
\frac{d \sigma}{d Y_{1} d k_{1}^{2} \ldots d Y_{n} d k_{n}^{2}} \sim \Phi^{T} G_{Y_{n}-Y_{0}}^{B F K L} L\left(k_{n}\right) \ldots G_{Y_{1}-Y_{2}}^{B F K L} L\left(k_{1}\right) G_{Y-Y_{1}}^{B F K L} \Phi^{P}
$$

The question we ask is how to generalize this expression when one of the colliding particles, the target, is large and dense. We then have to take into account the physics of gluon saturation, and associated non-linear evolution equations like BK-JIMWLK. The projectile's gluon scattering of a dense target has the eikonal propagator given by the Wilson line

$$
S(x)=\mathcal{P} \exp \left\{i \int d x^{-} T^{a} \mathbf{A}_{t}^{a}\left(x, x^{-}\right)\right\} .
$$

with $A_{t}$ characterizing the target external field. We find it convenient to introduce two targets - one for the amplitude $S$ and another one for its conjugate $\bar{S}$. In the end of our computation we set $S=\bar{S}$. The answer to our posed question has the following form

$$
\frac{d \sigma}{d Y_{1} d k_{1}^{2} \ldots d Y_{n} d k_{n}^{2}} \sim \int D S D \bar{S} W^{T}[S] \delta(S-\bar{S}) U_{Y_{n}-Y_{0}} \mathcal{O}_{g}^{k_{n}} \ldots U_{Y_{1}-Y_{2}} \mathcal{O}_{g}^{k_{1}} U_{Y-Y_{1}} \Sigma^{P}[S, \bar{S}]
$$

with the evolution operator

$$
U\left(Y_{1}-Y_{2}\right)=\operatorname{Exp}\left[-H_{3}\left(Y_{1}-Y_{2}\right)\right]
$$

Below we will present the Hamiltonian $H_{3}$ and the gluon emission vertex $\mathcal{O}_{g} . W^{T}$ and $\Sigma^{P}$ are generalized impact factors, which often appear in the color glass formalism. 
We first introduce the gluon production (and scattering) amplitude

$$
Q_{i}^{a}(z)=g \int_{x} \frac{(x-z)_{i}}{(x-z)^{2}}\left[J_{L}^{a}(x)-S^{a b}(z) J_{R}^{b}(x)\right]
$$

The generators of the left/right color rotations are Lie derivatives

$$
J_{R}^{a}(x)=-\operatorname{tr}\left\{S(x) T^{a} \frac{\delta}{\delta S^{\dagger}(x)}\right\}, \quad J_{L}^{a}(x)=-\operatorname{tr}\left\{T^{a} S(x) \frac{\delta}{\delta S^{\dagger}(x)}\right\}
$$

In terms of $Q$ the gluon emission operator which is found in Ref. [1] is

$$
\mathcal{O}_{g}^{k}[S, \bar{S}]=\int \frac{d^{2} z}{2 \pi} \frac{d^{2} \bar{z}}{2 \pi} e^{i k(z-\bar{z})} Q_{i}^{a}(z,[S]) Q_{i}^{a}(\bar{z},[\bar{S}])
$$

This operator is visualized in Fig. 2 The same operator $Q$ enters the expression for the Hamiltonian $H_{3}$ first introduced in Ref.[2]

$$
H_{3}[S, \bar{S}] \equiv \int_{z}\left[Q_{i}^{a}(z,[S])+Q_{i}^{a}(z,[\bar{S}])\right]^{2}
$$

As a first application of the above formalism we consider a single inclusive gluon production (Fig. 3).

Using our general formalism we write

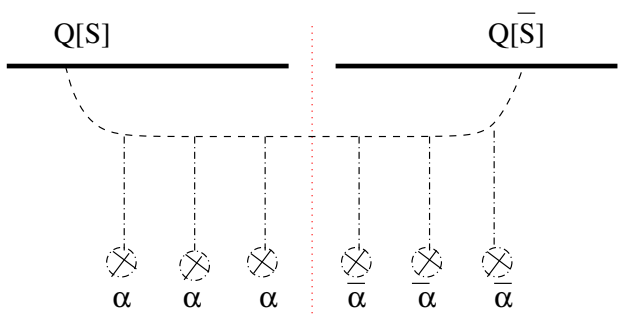
the cross section

$$
\frac{d \sigma}{d Y_{1} d k^{2}}=\int D S D \bar{S} W^{T}[S] \delta(\bar{S}-S) U_{Y_{1}} \mathcal{O}_{g}^{k} U_{Y-Y_{1}} \Sigma_{Y}^{P}
$$

This can be brought [1] to the following form [3]

$$
\begin{gathered}
\frac{d \sigma}{d Y_{1} d k^{2}}=\frac{\alpha_{s}}{\pi} \int_{z, \bar{z}} e^{i k(z-\bar{z})} \int_{x, y} \frac{(z-x)_{i}}{(z-x)^{2}} \frac{(\bar{z}-y)_{i}}{(\bar{z}-y)^{2}} G^{B F K L}\left(x, y ; Y-Y_{1}\right) \times \\
\times\left[\left\langle T_{z, y}\right\rangle_{Y_{1}}+\left\langle T_{x, \bar{z}}\right\rangle_{Y_{1}}-\left\langle T_{z, \bar{z}}\right\rangle_{Y_{1}}-\left\langle T_{x, y}\right\rangle_{Y_{1}}\right]
\end{gathered}
$$

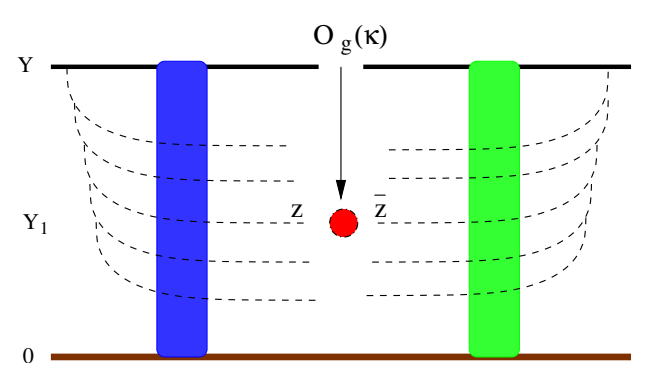

Figure 3: Single gluon production 
with $\langle T\rangle$ denoting the $S$-matrix of a gluonic dipole:

$$
\left\langle T_{x, y}\right\rangle_{Y_{1}} \equiv \int D S W_{Y_{1}}^{T}[S] \operatorname{tr}\left[S_{x}^{\dagger} S_{y}\right]
$$

which can be deduced from solutions of the BK-JIMWLK equations. Our solution for the multi-gluon production problem is given in terms of Feynman-like diagrammatic technique. Fig. 4 is an example of a diagram for the one gluon case.

We were not able to proceed with the general formalism beyond the one gluon case. Instead we had to rely on the dipole approximation. In practice we introduce new degrees of freedom and re-express both the Hamiltonian $\mathrm{H}_{3}$ and the vertex $\mathcal{O}_{g}$ in the new degrees. The dipole creation operator reads (similarly $\bar{s}$ )

$$
s_{x, y}=\frac{1}{N} \operatorname{tr}\left[S_{F}(x) S_{F}^{\dagger}(y)\right]
$$

We also find it necessary to introduce the quadrupole operator

$$
q_{x, y, u, v}=\frac{1}{N} \operatorname{tr}\left[S_{F}(x) S_{F}^{\dagger}(y) S_{F}(u) S_{F}^{\dagger}(v)\right] .
$$

It is important to stress that no other higher multiplet

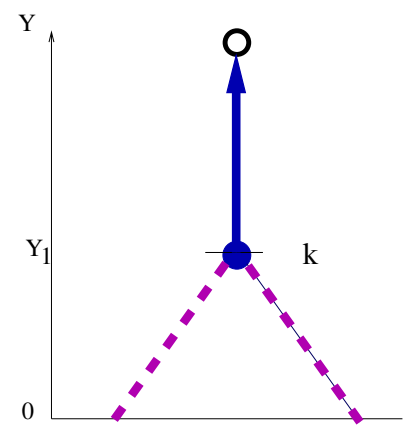

Figure 4: Single gluon production: Diagrammatic representation operators is needed if the projectile at rest is made only out of dipoles. Furthermore, the quadrupoles of the mixed type arise

$$
q_{x, y, v, u}^{s \bar{s}}=\frac{1}{N} \operatorname{tr}\left[S_{F}(x) S_{F}^{\dagger}(y) \bar{S}_{F}(u) \bar{S}_{F}^{\dagger}(v)\right]=q_{x, y, v, u}+t_{x, y, v, u}
$$

Note that we have to set $\bar{S}=S$ at the end of our computation $t=0$. That leads us to a perturbation theory in $t$.

Re-express the Hamiltonian $H_{3}$ in new degrees of freedom we find four terms:

$$
H_{3}=H_{s}+H_{q}+H_{t}+V_{t \rightarrow t t}
$$

$H_{s}$ is the dipole Hamiltonian which generates the BK eq. for the dipole $s$ :

$$
\partial_{y} s(x, y)=K^{B F K L} \otimes(s-s s)
$$

$H_{q}$ generates a linear evolution of $q$ (similar to BKP) which is also coupled to the external field $s$ :

$$
\partial_{y} q(x, y, u, v)=K_{1} \otimes q+K_{2} \otimes q s+K_{3} \otimes s s
$$

The explicit expressions for the kernels $K$ can be found in [1]. $H_{t}$ generates a linear evolution of $t$ which is also coupled to $s$ :

$$
\partial_{y} t(x, y, u, v)=G^{-1}[s] \otimes t+\lambda \otimes t t
$$

Here $G$ is a propagator in the external Pomeron field $s$. When $x=v$ and $y=u$, the twopoint function $G$ coincides with $G^{B F K L}$. The propagator $G$ and triple $t$ vertex $\lambda$ are in the basic of our perturbation theory. They define the relevant Feynman rules (Fig. 5). 
Finally we have to re-express the insertion operator $\mathcal{Q}_{g}$ in terms of the new degrees of freedom. We find that $\mathcal{O}_{g}$ splits into three groups of vertecies (Fig. 6):

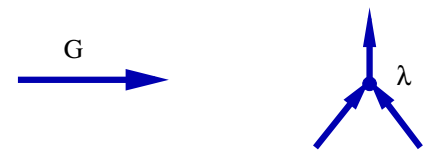

Figure 5: The propagator and vertex

$$
\mathcal{O}_{g}(k)=A_{-1}(k)+A_{0}(k)+A_{1}(k)
$$

The first group reduces the number of $t^{\text {'s }}$ by one; the second group leaves the number unchanged, the last group increases the number of $t$ 's by one. Remember that $t$ is set to zero at the end. So any diagram which has an external line propagating $t$ is zero. Fig. 7 presents an example of our diagram technique as applied to double gluon emission. Our expressions reproduce the result of Ref. [4].

\section{Acknowledgments}

Everything which is reported above has been done in collaboration with Alex Kovner. Many thanks to Alex for making hard work into a joy.

\section{References}

[1] A. Kovner and M. Lublinsky, JHEP 0611083 (2006); A. Kovner, M. Lublinsky and H. Weigert, Phys. Rev. D74 114023 (2006).

[2] M. Hentschinski, H. Weigert and A. Schafer, Phys. Rev. D73 051501 (2006).

[3] Y. V. Kovchegov and K. Tuchin, Phys. Rev. D65 074026 (2002).

[4] J. Jalilian-Marian and Y. V. Kovchegov, Phys. Rev. D70 114017 (2004).

[5] Slides: http: //indico. cern. ch/contributionDisplay $\cdot$ py? contribId=51\&sessionId=8\& conf $I d=9499$

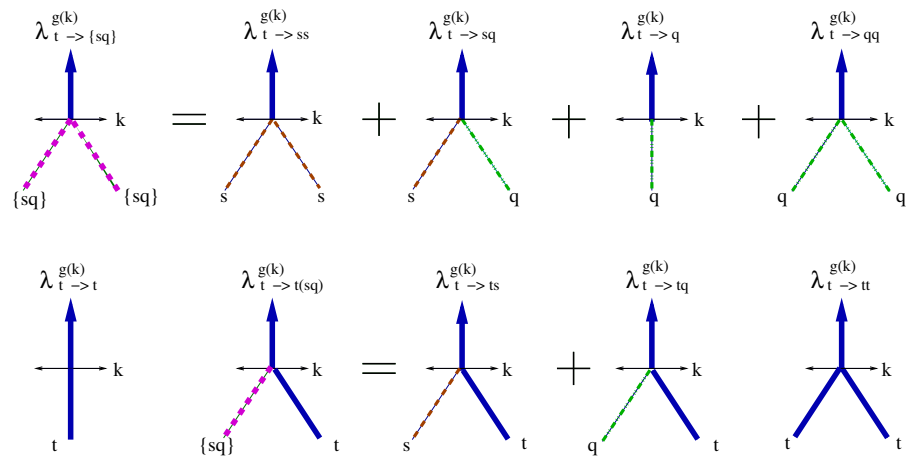

Figure 6: The gluon emission vertices in the dipole approximation

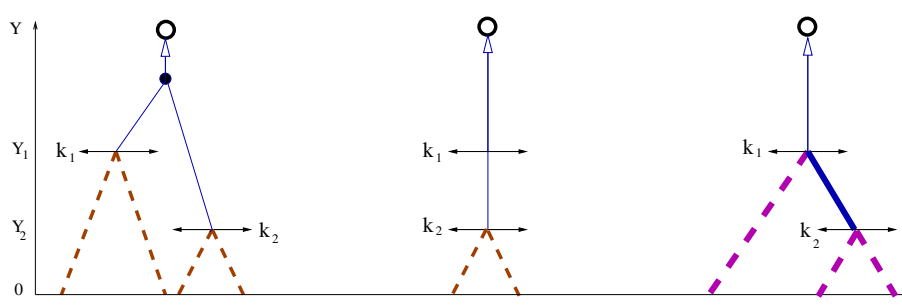

(a)

(b)

(c)

Figure 7: Double-gluon production 